\title{
OPORTUNIDADES DE APRENDIZAGEM COM APOIO DA COMUNICAÇÃO AUMENTATIVA E ALTERNATIVA EM TEMPOS DE COVID-19
}

\author{
OPORTUNIDADES DE APRENDIZAJE CON APOYO DE COMUNICACIÓN \\ AUMENTATIVA Y ALTERNATIVA EN TIEMPOS DE COVID-19
}

\author{
LEARNING OPPORTUNITIES WITH AUGMENTATIVE AND ALTERNATIVE \\ COMMUNICATION SUPPORTS IN TIMES OF COVID-19
}

\author{
Renata BONOTTO ${ }^{1}$ \\ Ygor CORRÊA ${ }^{2}$ \\ Eduardo CARDOSO ${ }^{3}$ \\ Daianne Serafim MARTINS ${ }^{4}$
}

RESUMO: Este estudo analisa os conteúdos de postagens envolvendo Comunicação Aumentativa e Alternativa (CAA) na página ComunicaTEA_pais no Instagram, para identificar que materiais estão sendo criados e disponibilizados para apoiar a aprendizagem de estudantes com necessidades complexas de comunicação (NCC) durante o fechamento das escolas devido à pandemia pelo novo coronavírus. A partir da técnica de Análise de Conteúdo, esta pesquisa qualitativa exploratória analisou 94 postagens. Cinco categorias se destacaram, a saber: orientações sobre a Covid-19; atividades educativas formais; atividade educativas não formais; orientações sobre o uso de CAA e recursos tecnológicos. Os conteúdos das postagens revelam a contribuição da página para difundir informações e atenuar desigualdades ao promover acesso a recursos de apoio à comunicação. Constata-se um processo dinâmico de apoio educacional e psicossocial para a comunidade de pessoas com NCC e sua rede de apoio frente às mudanças na rotina escolar de alunos com deficiência e NCC.

PALAVRAS-CHAVE: Comunicação aumentativa e alternativa. COVID-19. Materiais educacionais acessíveis. Necessidades complexas de comunicação.

RESUMEN: Este estudio analiza el contenido de las publicaciones que involucran la Comunicación Aumentativa y Alternativa (CAA) en la página ComunicaTEA pais en Instagram para identificar qué materiales se están creando y poniendo a disposición para apoyar el aprendizaje de los estudiantes con necesidades de complejas de comunicación

\footnotetext{
${ }^{1}$ Alternativa Inclusiva (AI), Porto Alegre - RS - Brasil. Consultora e Formadora em Comunicação Aumentativa e Alternativa. Doutorado em Informática na Educação (FURG). ORCID: https://orcid.org/0000-0003-42716042. E-mail: bonotto.renata@gmail.com

${ }^{2}$ Universidade de Caxias do Sul (UCS) Caxias do Sul - RS - Brasil. Pós-Doutorando Programa de PósGraduação em Educação. ORCID: https://orcid.org/0000-0002-3526-9195. E-mail: correaygorprof@gmail.com

${ }^{3}$ Universidade Federal do Rio Grande do Sul (UFRGS), Porto Alegre - RS - Brasil. Professor Adjunto do Departamento de Design e Expressão Gráfica e Professor Colaborador do Programa de Pós-Graduação em Design. Doutorado em Design (UFRGS). ORCID: https://orcid.org/0000-0002-1202-1779. E-MAIL: eduardo.cardoso@ufrgs.br

${ }^{4}$ Universidade Federal do Rio Grande do Sul (UFRGS), Porto Alegre - RS - Brasil. Doutoranda do Programa de Pós-graduação em Design. ORCID: https://orcid.org/0000-0002-7918-7693. E-mail: to.daianne@gmail.com
}

RIAEE - Revista Ibero-Americana de Estudos em Educação, Araraquara, v. 15, n. 4, p. 1730-1749, out./dez. 2020. e-ISSN: $1982-5587$. 
(NCC) durante el cierre de las escuelas debido a la pandemia causada por el nuevo coronavirus. Basado en la técnica de Análisis de Contenido, esta investigación exploratoria cualitativa analizó 94 publicaciones. Se destacaron cinco categorías, a saber: directrices sobre Covid-19; actividades educativas formales; actividades educativas no formales; orientación sobre el uso de CAA y recursos tecnológicos. El contenido de las publicaciones revela la contribución de la página para difundir información y mitigar las desigualdades al promover el acceso a recursos para apoyar la comunicación. Se percibe un proceso dinámico de apoyo educativo y psicosocial para la comunidad de personas con NCC y su red de apoyo ante los cambios en la rutina escolar de los estudiantes con discapacidades y NCC.

PALABRAS CLAVE: Comunicación aumentativa y alternativa. COVID-19. Materiales educativos accesibles. Necesidades complejas de comunicación.

ABSTRACT: This study analyzes the content of posts involving Augmentative and Alternative Communication (AAC) on Instagram by ComunicaTEA_pais page to identify what materials are being created and made available to support the learning of students with complex communication needs (CCN) during the closing of schools due to the pandemic caused by the new coronavirus. Based on the Content Analysis technique, this exploratory qualitative research analyzed 94 posts. Five categories stood out, namely: guidelines on Covid-19; formal educational activities; non-formal educational activities; guidance on the use of AAC and technological resources. The contents of the posts reveal the contribution of the page in providing information to mitigate inequalities by promoting access to resources to support communication. There is a dynamic process of educational and psychosocial support for the community of people with CCN and their support network in face of changes in the school routine of students with disabilities and CCN.

KEYWORDS: Augmentative and alternative communication. COVID-19. Accessible educational materials. Complex communication needs.

\section{Introdução}

O presente estudo investiga as postagens realizadas na página da rede social Instagram intitulada ComunicaTEA_pais ${ }^{5}$, durante o período de fechamentos das escolas no Brasil, devido à pandemia de Covid-196, visando a identificar que materiais estão sendo criados e disponibilizados para apoiar a aprendizagem de estudantes com necessidades complexas de comunicação (NCC). As NCCs decorrem de transtornos do desenvolvimento ou condições adquiridas que impactam a fala e a comunicação. A página ComunicaTEA_pais se dedica à

${ }^{5}$ ComunicaTEA_pais é uma iniciativa de pais de crianças com NCC voltada à difusão de conhecimentos sobre CAA.

6 "COVID-19 é causada pelo agente etiológico nomeado SARS-CoV-2, a qual surgiu, inicialmente, na China, em novembro de 2019 e se espalhou pelo país e pelo mundo" (CASTAMAN; RODRIGUES, 2020, p. 03). 
difusão de conhecimento sobre Comunicação Aumentativa e Alternativa ${ }^{7}$ (CAA) e materiais de apoio acessíveis para pessoas com necessidades complexas de comunicação (NCC) e sua rede de apoio. Durante a pandemia de Covid-19, a página tem se destacado no acolhimento das famílias, dos professores, dos profissionais e dos alunos com NCC, no momento de distanciamento social, que teve início, oficialmente em 17 de março de 2020 no Brasil. Ao considerar que o Estado brasileiro é signatário da Convenção Internacional sobre os Direitos da Pessoa com Deficiência (CDPD) (ONU, 2007), o direito à educação está formalmente assegurado por leis e políticas no país para as pessoas com deficiência (PcD) (MEC, 2016). Nesse momento, contudo, os esforços têm sido no sentido de evitar o apagamento da PcD (PRATES, 2020a) e o aprofundamento da violação dos seus direitos ainda mais diante da pandemia de COVID-19. Esta é uma pesquisa qualitativa de natureza exploratória (SAMPIERI; COLLADO; LUCIO, 2013), na qual se investigou os conteúdos das postagens realizadas na página ComunicaTEA_pais no Instagram durante o período de 10 março à 31 maio de 2020, contabilizando um total de 174 postagens. Desse modo, conforme anteriormente exposto, tece-se como propósito da pesquisa identificar que materiais estão sendo disponibilizados para apoiar a aprendizagem de estudantes com NCC nesse momento de isolamento e Ensino Remoto Emergencial (WILLIAMSON; EYNON; POTTER, 2020).

\section{Educação de estudantes com deficiência em tempos de Covid-19}

A partir do anúncio da pandemia mundial pelo COVID-19 pela Organização Mundial da Saúde (OMS) (CASTAMAN; RODRIGUES, 2020), assim como em outros países, no Brasil, a pandemia demandou o estabelecimento de estratégias de distanciamento social por tempo indeterminado ${ }^{8}$ (VILLAS BÔAS; UNBEHAUM, 2020).O fechamento das instituições educacionais se deu por meio do decreto das Portarias $\mathrm{N}^{\circ} 343$, de 17 de março de 2020 (BRASIL, 2020a) e No 544, de 16 de junho de 2020 (BRASIL, 2020b) e da Medida Provisória $N^{o}$ 934, de $1^{\circ}$ de abril de 2020 (BRASIL, 2020c), os quais definiram a substituição da modalidade de aulas presenciais pela modalidade de Ensino Remoto Emergencial (ERE) (WILLIAMSON; EYNON; POTTER, 2020), possivelmente, até o mês de dezembro de 2020 (SANTOS, 2020).

\footnotetext{
${ }^{7}$ Há outras formas de referência ao campo no Brasil como Comunicação Ampliada e Alternativa e Comunicação Suplementar e Alternativa. Optamos pela denominação Comunicação Aumentativa e Alternativa pela maior proximidade com o termo adotado pela comunidade internacional em inglês.

${ }^{8}$ Até a data da submissão deste artigo a quarentena estava em vigor, ou seja, início do mês de julho de 2020.
} 
Segundo a UNESCO (2020), com o fechamento de escolas e universidades, no mundo, aproximadamente 1,2 bilhão de estudantes ficam sem aulas em virtude dos efeitos da pandemia de Covid-19 e, consequentemente, desatendidos por suas instituições em um primeiro momento, de maneira que os processos educacionais passaram a ocorrer na modalidade de ensino domiciliar (ZHAO, 2020). O cenário educacional contemporâneo já vinha operando, em certa medida, na lógica do Bring Your Own Device (BYOD) (do inglês: Traga Seu Próprio Dispositivo), ou seja, o aluno leva seu dispositivo para a escola. A partir da COVID-19, no entanto, entra em vigor a lógica Bring Your Own School Home (BYOSH) (do inglês: Traga sua Própria Escola para Casa), haja visto o deslocamento das atividades educacionais para o âmbito domiciliar (WILLIAMSON; EYNON; POTTER, 2020). De acordo com Williamson, Eynon e Potter (2020), o ERE tem causado grandes mudanças para todos os atores envolvidos na cena educacional, quer sejam esses professores (produção de conteúdo, aulas, avaliação), alunos (realização de atividades) ou famílias (auxílio na execução das atividades). De acordo com Moreira e Schlemmer (2020), no ERE, as aulas online são dotadas da mesma constituição das aulas presenciais evidenciando uma transposição equivocada do formato presencial para o online com a mediação de recursos tecnológicos digitais, tais como computadores desktop, notebooks, tablets e smartphones ligados à web pela Internet. Assim, diante do cenário de isolamento social, as instituições de ensino e profissionais da educação atribuíram grande potência ao uso das Tecnologias Digitais.

A orientação do governo brasileiro sobre a continuidade do processo educacional via ERE, evidenciou, mais uma vez, um abismo entre a distinção de classes sociais no Brasil, posto que muitas escolas da rede de Educação Básica, municipais e estaduais, assim como instituições de Ensino Superior não possuem condições de infraestrutura para dar seguimento às aulas online (NÓVOA, 2020). As Tecnologias Digitais Interativas (GARCIA et al., 2011, p. 82), em meio ao caos, obtiveram maior emprego porque possibilitam a mediação de diversas atividades via ERE. Por outro lado, demandaram que professores tivessem que ressignificar suas práticas de ensino e aprendizagem no contexto da pandemia. Deste modo, os processos didático-pedagógicos na versão online, mesmo que temporariamente, têm exigido que os professores repensem o modo como os alunos podem aprender e ser avaliados remotamente.

Castaman e Rodrigues (2020, p. 3) enfatizam que o distanciamento social em relação ao cenário educacional tem sido um agravante, no que se refere à educação, a saber pelo fato de que esse vem "agravando inclusive a evasão e o aumento da desigualdade, assim como o desconforto de ter que assumir o processo de ensino e aprendizagem como condição de 
autonomia, de empoderamento e de autodeterminação". Diante desse cenário, cabe problematizar também, os desdobramentos para a educação de estudantes com deficiência que, historicamente, já experimentam várias barreiras, e, cujo direito à educação fica ainda mais fragilizado em momentos de crise (WORLD BANK, 2019).

\section{A educação de pessoas com deficiência}

A educação é um direito universal e, nas últimas décadas, a compreensão da necessidade de garantia do direito à educação para as pessoas com deficiência têm sido constantemente reiterado nos âmbitos internacional e nacional. Internacionalmente, um dos documentos mais importantes da atualidade é a Convenção Internacional sobre os Direitos da Pessoa com Deficiência (CIDPD) (ONU, 2007), que foi promulgada no Brasil pelo Decreto Legislativo ${ }^{\circ} 186$ e Decreto ${ }^{\circ} 6.949$ de 25 de agosto de 2009.

Uma das premissas da CIDPD no seu artigo $1^{\circ}$ é "promover, proteger e assegurar o exercício pleno e equitativo de todos os direitos humanos e liberdades fundamentais por todas as pessoas com deficiência e promover o respeito pela sua dignidade inerente" (ONU, 2007, $\mathrm{s} / \mathrm{p}$ ). Além do tema Educação, que é tratado ao longo do artigo ${ }^{\circ} 24$, é importante considerar o artigo 9, sobre Acessibilidade, quando fica destacada a necessidade de medidas para assegurar o acesso em condições de igualdade com as demais, ao meio físico, aos transportes, à informação e comunicações, incluindo os sistemas e as tecnologias da informação e de comunicação, pois só assim será possível que as pessoas com deficiência vivam com independência e participem plenamente em todos os aspectos da vida (BRASIL, 2009). Além da CDPD, leis infraconstitucionais e outros marcos legais reafirmam o direito à educação dos estudantes com deficiência, dentre as mais recentes, a Lei Brasileira de Inclusão (BRASIL, 2015) e o Plano Nacional de Educação (BRASIL, 2014), que define metas específicas concernentes à educação das pessoas com deficiência.

Esforços têm sido empreendidos para evitar o apagamento da pessoa com deficiência (PRATES, 2020a) e a violação dos seus direitos, ainda mais diante da pandemia de COVID19, que impõe o distanciamento social e, por isso, tem o potencial de aprofundar processos de segregação e exclusão já que pessoas com deficiência figuram como sujeitos dos grupos de risco para contaminação pelo vírus da COVID-19 (PRATES, 2020b). De forma concreta, em uma pesquisa no site ${ }^{9}$ do Ministério da Educação não são identificadas quaisquer orientações, ações ou documentos norteadores em prol da educação de estudantes com deficiência e

\footnotetext{
${ }^{9}$ Acompanhamento da atualização das informações no site do MEC até o dia 30 de junho de 2020.
} 
necessidades complexas de comunicação até então. Contudo, para a efetivação do direito à educação é importante a identificação e a eliminação de barreiras à aprendizagem, à comunicação bem como a provisão de adaptações razoáveis ${ }^{10}$, ou seja, medidas de apoio individualizadas. As adaptações e medidas de apoio individualizadas remetem à tecnologia e à CAA como basilares nos processos educativos, em especial, para os estudantes com deficiência com NCC.

\section{Comunicação Aumentativa e Alternativa (CAA)}

A tecnologia desempenha um papel crucial na promoção de autonomia e participação social livre de barreiras para a pessoa com deficiência. A CAA, sendo uma subárea da Tecnologia Assistiva, se alinha aos objetivos de promover a autonomia e a participação enquanto colabora para a eliminação das barreiras de comunicação e das barreiras de acesso à informação (ONU, 2007; BONOTTO, 2016) para as pessoas experimentando condições que impactam a fala, quer restringindo ou impedindo-a de forma temporária ou permanente (BUEKELMAN; LIGHT, 2020). Ao pensar sobre a educação de pessoas com deficiência, Vigotski (2011) destaca as possibilidades quanto ao desenvolvimento cultural onde o caminho do desenvolvimento natural encontra obstáculos. O caminho do desenvolvimento cultural diz respeito ao conhecimento socialmente constituído e organizado para intervir e modificar através de "estratégias artificiais" o meio em que vivemos (VIGOTSKI, 2011). Postula-se aqui que a CAA pode atuar como estratégia de acesso ao arcabouço da comunicação (BONOTTO, 2016).

O campo da Comunicação Aumentativa e Alternativa (CAA) diz respeito à concepção, ao desenvolvimento, à produção e à distribuição de assistência e apoios. De acordo com a ASHA (2004), a CAA consiste em um conjunto integrado de símbolos, recursos, técnicas e estratégias. Os símbolos constituem unidades representacionais, que podem envolver gestos, imagens ou sons, que representam palavras ou mensagens. Os recursos podem ser materiais impressos, físicos ou eletrônicos, envolvendo o uso de dispositivos, como celulares, tablets e computadores (ASHA, 2004). Um formato de recurso de CAA muito utilizado são as pranchas de comunicação, que são grades de linhas e colunas onde cada quadro pode conter uma imagem (símbolo) e texto, ou seja, o recurso contém os símbolos que viabilizam a construção de mensagens para mediar a comunicação. As técnicas e estratégias dizem respeito

10 "as modificações e os ajustes necessários e adequados... quando requeridos em cada caso, a fim de assegurar que as pessoas com deficiência possam gozar ou exercer, em igualdade de oportunidades com as demais pessoas, todos os direitos humanos e liberdades fundamentais" (ONU, 2007). 
a como os recursos são acessados e as mensagens podem ser transmitidas com mais rapidez e eficiência no processo comunicacional.

Beukelman e Light (2020) destacam que o propósito central da intervenção com a CAA não é encontrar uma solução tecnológica para problemas de comunicação, mas habilitar indivíduos para, de maneira eficiente e eficaz, engajarem-se em uma gama variada de interações e participarem em atividades de sua escolha, exercendo assim, autodeterminação. De modo mais específico, essas interações comunicativas permitem (1) comunicar necessidades e vontades; (2) transferir informações; (3) aproximar socialmente para estabelecer, manter ou desenvolver engajamento social para construir relacionamentos; (4) praticar etiqueta social e, (5) proporcionar organização por meio de diálogo interno (BEUKELMAN; LIGHT, 2020).

Os benefícios da CAA estão bem estabelecidos na literatura, a CAA (1) aprimora a comunicação, (2) apoia o desenvolvimento da linguagem, (3) aumenta a participação, (4) apoia a compreensão e (5) diminui a frustração e os problemas de comportamento. Uma vez que a comunicação é essencial para a mediação da aprendizagem e do desenvolvimento, para efetivar o direito à educação de estudantes com deficiência com necessidades complexas de comunicação, a CAA fornece à pessoa a possibilidade de autonomia e de acessar as oportunidades em condição de igualdade com seus pares (BEUKELMAN; LIGHT, 2020).

\section{Método}

Esta é uma pesquisa ${ }^{11}$ qualitativa (SAMPIERI; COLLADO; LUCIO, 2013) de caráter exploratório, haja vista o pouco conhecimento sobre o papel das redes sociais no estabelecimento de estratégias de enfrentamento da Covid-19 no período de distanciamento social devido à pandemia e seus impactos na educação.

O problema de pesquisa norteador desse estudo foi a identificação dos tipos de conteúdos das postagens disponibilizadas pela página ComunicaTEA_pais para apoiar estudantes com NCC, nesse momento de ERE. A escolha da página ComunicaTEA_pais se deu por conveniência pois é a página sobre o tema no Instagram com a maior regularidade nas postagens, maior número de inscritos (atualmente, mais de 10.000) e maior diversidade de materiais. De acordo com as informações obtidas no perfil, a página ComunicaTEA_pais é um grupo formado por pais de crianças no espectro do autismo com NCC sendo uma

${ }^{11}$ Pesquisa baseada em materiais disponíveis de modo público na rede social, não constituindo pesquisa com seres humanos e, portanto, não requerendo aprovação em comitê de ética para sua execução (KOZINETS, 2014). 
iniciativa ligada ao grupo de estudos ComunicaTEA ${ }^{12}$. O período de registro das postagens foi de 10 de março a 31 de maio de 2020. Esse recorte temporal se justifica por coincidir com o momento de constatação da pandemia e do decreto de paralisação da oferta de ensino presencial e estabelece o ERE, que até a finalização desse estudo, não havia chegado ao fim.

Para o tratamento dos dados, adotou-se a técnica de Análise de Conteúdo (AC) proposta de Bardin (2011) aos conteúdos em imagem, texto e vídeo do corpus, abrangendo três etapas, a saber, (a) pré-análise, (b) exploração do material e (c) tratamento dos resultados. De acordo com Bardin (2011), a etapa de pré-análise compreende a leitura "flutuante", a partir da qual, ocorre a escolha dos documentos, a formulação das hipóteses, a referenciação e a elaboração dos índices e indicadores, portanto, a preparação dos materiais que serão submetidos análise. A etapa de exploração do material compreende a análise propriamente dita - é o momento da aplicação sistemática de decisões. Nesta etapa, se dá a codificação que emerge da análise aliada à classificação e à categorização dos dados; e, também, é o momento que leva à percepção de homogeneidade, pertinência, objetividade, fidelidade e produtividade dos dados por um lado e a exclusão de dados não pertinentes. Por fim, a última etapa é a de tratamento dos resultados, onde são ressaltados os dados significativos e válidos (BARDIN, 2011).

\section{Resultados e discussão}

O processo de análise do corpus de pesquisa deu-se por meio das etapas de préanálise, exploração do material e tratamento dos resultados, os quais foram desdobradas para integrar cinco fases que compõem a técnica de Análise de Conteúdo (BARDIN, 2011). Por meio da leitura "flutuante" (BARDIN, 2011), foi realizado o primeiro contato com o conteúdo de 174 postagens da página ComunicaTEA_pais realizadas entre os dias 10 de março e 31 de maio de 2020. Algumas hipóteses foram formuladas sobre as possíveis contribuições educacionais acerca da variada gama de temas nas postagens, a saber, sugestões de livros, dicas de materiais variados, receitas, atividades e eventos relacionados à CAA. Desta forma, iniciou-se também o descarte de postagens que não ofereciam uma contribuição educacional

${ }^{12}$ ComunicaTEA conta com o apoio do grupo do Ambulatório de Cognição Social Dr. Marcos T. Mercadante (TEAMM) do Departamento de Psiquiatria da Universidade Federal de São Paulo (UNIFESP). O objetivo do ComunicaTEA é "fomentar os estudos e divulgação da Comunicação Aumentativa e Alternativa no Brasil". Sua trajetória tem início em abril de 2019 com a participação de um evento e posterior estabelecimento de um grupo de estudos ligado ao TEAMM sobre CAA, com reuniões presenciais mensais com início em agosto de 2019, agregando além de famílias, profissionais como psicólogas, terapeutas ocupacionais, fonoaudiólogas, educadoras, etc. 
mais diretamente visível como as dicas de livros sobre CAA, a divulgação de eventos e as frases de especialistas, ou seja, as instâncias em que havia referência ao campo da CAA, mas não necessariamente a utilização aplicada da mesma.

A etapa de pré-análise correspondeu à Fase 1, Preparação dos Dados, para análise das postagens realizadas no escopo de tempo definido, ou seja, entre os dias 10 de março e 31 de maio de 2020. Das 32 postagens realizadas na página em março, 10 atenderam ao escopo deste estudo; das 67 postagens em abril, 38; e das 75 postagens de maio, 46. Assim, para a análise de dados, foram consideradas 94 postagens da página ComunicaTEA_pais. As postagens foram registradas em uma planilha por ordem cronológica de publicação, tema, descrição e mídia utilizada. Cada postagem recebeu o código $(\mathrm{P})$ seguido de um número correspondente à ordem de registro, como, por exemplo, P01 - Postagem 01, de modo que todos os códigos elaborados foram registrados em uma planilha de Excel, contendo também data (dia/mês/ano), tema e formato (texto, imagem ou vídeo).

A etapa de exploração do material correspondeu às Fase 2 - Exploração do material; Fase 3 - Codificação; e Fase 4 Categorização. Na Fase 2, Exploração do material, as postagens, anteriormente, ordenadas foram novamente tabuladas, a fim de proceder à codificação. Nesse momento, as postagens foram agregadas por tipo e semelhança (materiais relacionados à COVID-19, brincadeiras, jogos, organizadores da rotina, música, histórias adaptadas etc.). As próprias formas de apresentação dos materiais das postagens também favoreciam esse agrupamento, por exemplo, Dica de material - Jogos, Dica de material Receitas, Dica de materiais. Na Fase 3, Codificação, foram criados códigos, de modo a operacionalizar o processo de categorização do tema das postagens. Posteriormente, realizouse uma leitura crítica dos conteúdos das postagens e foram criados códigos que expressam a finalidade em CAA do tema de cada postagem, perfazendo um total de 13 códigos.

Na Fase 4, Categorização, a presente etapa tem como objetivo a união, ou seja, a formação de conjuntos de códigos por incidência e semelhança, ação que permite agrupar dados e consolidar um significado para tais informações. Depois que todas as postagens foram codificadas e categorizadas, tratou-se de mapear os códigos que tiveram maior incidência e semelhança diante dos tipos de temas das postagens. Dessa fase, emergiram cinco grandes grupos de postagens: postagens sobre COVID-19, postagens com atividades educativas não formais, postagens com atividades educativas formais, postagens com orientações sobre o uso da CAA e Recursos Tecnológicos. Por fim, a etapa 3 de tratamento dos resultados corresponde à Fase 5, a Análise de Conteúdo propriamente dita (BARDIN, 2011). Nessa fase, os códigos com maior incidência e semelhança foram submetidos a quatro procedimentos 
adicionais, por meio da Análise de Conteúdo (BARDIN, 2011), sendo esses: a) leitura detalhada; b) análise reflexiva, por parte dos pesquisadores, acerca do tema e finalidade de cada postagem; c) identificação e criação dos grupos de categorias de convergência, para fins de consolidação de um significado; e d) criação de categorias de significados. Cinco categorias emergiram a partir das incidências e semelhança entre os conteúdos presentes nas postagens: COVID-19, orientação sobre o uso da CAA, atividades educativas não formais, atividades educativas formais e recursos tecnológicos.

Foram categorizadas como atividades educativas formais aquelas postagens que continham materiais que poderiam favorecer o acesso ao currículo escolar como a leitura em livros adaptados e conteúdos de português, matemática, ciências humanas e ciências naturais. As atividades com potencial de favorecer a organização das dinâmicas familiares e a ocupação do tempo de forma produtiva para pais e filhos durante o período distanciamento social, como receitas, músicas, rotinas, jogos e brincadeiras foram categorizadas como atividades educativas não formais.

O Quadro 1 sintetiza o resultado dos dados gerados nas fases 2, 3 e 4 por ordem de semelhança e incidência das categorias e códigos correspondentes: (a) Atividade Educativa não Formal (46 postagens) com 4 códigos - jogos e brincadeiras, receitas, rotinas diárias e músicas; (b) Orientação sobre o uso de CAA (16 postagens) com 2 códigos - mediação comunicativa e organizadores comportamentais; (c) Atividade Educativa Formal (15 postagens) com dois códigos - conteúdos acadêmicos e leitura (d) COVID-19 (10 postagens) com três códigos - informação para evitar o contágio, pranchas de CAA sobre o estado de saúde e caracterização da COVID-19; e, por fim, (e) Recursos Tecnológicos (06 postagens) com 2 códigos - aplicativos em geral e aplicativos de CAA. Destaca-se que as cinco postagens sob o código Aplicativos em geral continham indicações de 4 a 5 aplicativos que se assemelhavam às definições das categorias Atividade educativa formal e Atividade educativa não formal, contudo, por estarem reunidos de modo aleatório, optou-se por considerá-los com uma categoria em separado pois a unidade de análise foi a postagem. 
Tabela 1 - Síntese de dados coletados por ordem de semelhança e incidência das categorias e códigos correspondentes

\begin{tabular}{|c|c|c|c|c|}
\hline Categoria & $\begin{array}{l}\text { Número de } \\
\text { Incidências }\end{array}$ & & Códigos & $\begin{array}{l}\text { Número de } \\
\text { Incidências }\end{array}$ \\
\hline \multirow{4}{*}{$\begin{array}{l}\text { Atividade } \\
\text { Educativa Não- } \\
\text { formal }\end{array}$} & \multirow{4}{*}{46} & AENF-JB & Jogos e brincadeiras & 15 \\
\hline & & AENF-R & Receitas & 13 \\
\hline & & AENF-RD & Rotinas diárias & 11 \\
\hline & & AENF-M & Músicas & 08 \\
\hline \multirow{2}{*}{$\begin{array}{l}\text { Orientação } \\
\text { sobre } \\
\text { o uso da CAA }\end{array}$} & \multirow[b]{2}{*}{16} & OR-MC & Mediação comunicativa & 12 \\
\hline & & OR-OC & $\begin{array}{c}\text { Organizadores } \\
\text { comportamentais }\end{array}$ & 04 \\
\hline \multirow{2}{*}{$\begin{array}{l}\text { Atividade } \\
\text { Educativa } \\
\text { Formal }\end{array}$} & \multirow[b]{2}{*}{15} & AEF-CA & Conteúdos acadêmicos & 09 \\
\hline & & AEF-L & Leitura & 06 \\
\hline \multirow{3}{*}{ Covid-19 } & \multirow{3}{*}{10} & CV-CEC & $\begin{array}{c}\text { Orientação para evitar } \\
\text { contágio }\end{array}$ & 05 \\
\hline & & CV-PES & $\begin{array}{l}\text { Pranchas de CAA sobre } \\
\text { estado de saúde }\end{array}$ & 03 \\
\hline & & $\mathrm{CV}-\mathrm{CC}$ & $\begin{array}{c}\text { Caracterização da } \\
\text { Covid-19 }\end{array}$ & 02 \\
\hline \multirow{2}{*}{$\begin{array}{l}\text { Recursos } \\
\text { Tecnológicos }\end{array}$} & \multirow[b]{2}{*}{06} & RT-APG & Aplicativos em geral & 05 \\
\hline & & RT-APCAA & Aplicativos de CAA & 01 \\
\hline
\end{tabular}

Fonte: elaborado pelos autores

A categoria Atividade Educativa Não Formal foi aquela com a maior incidência de códigos e números de postagens (46/94) e englobou recursos de CAA envolvendo Jogos e Brincadeiras, Receitas, Rotinas Diárias e Música. A Figura 1 apresenta um exemplo de aplicação com o código mais recorrente: Jogos e Brincadeiras. 
Figura 1 - Exemplo da categoria Jogos e Brincadeiras.

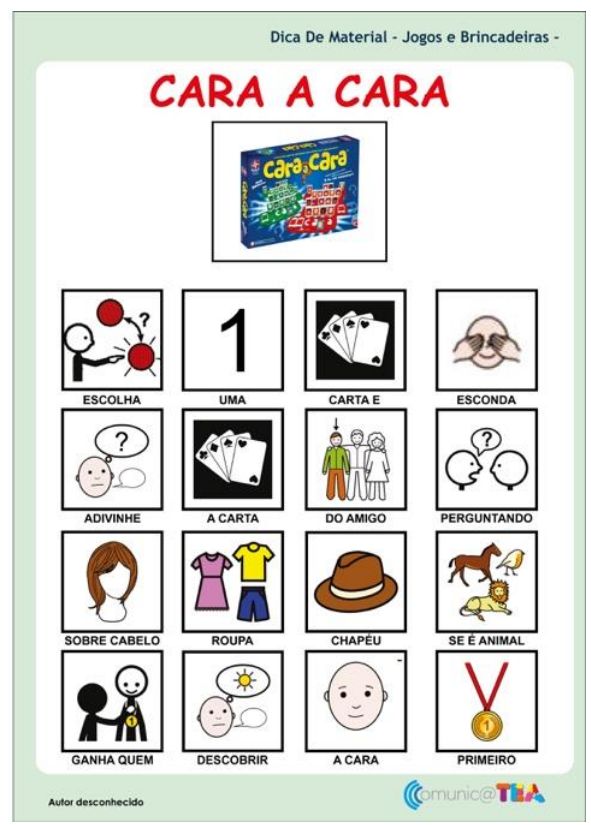

(Descrição da Imagem) Imagem vertical da página ComunicaTEA_pais. Sobre fundo branco 17 símbolos, sendo um no topo e maior e 16 logo abaixo e divididos em 4 linhas e 4 colunas. Acima do primeiro símbolo, em vermelho, o texto: CARA A CARA. Abaixo de cada símbolo uma palavra em preto. Ao redor uma borda verde clara com o texto em azul escuro na parte superior: Dica de Material: jogos e brincadeiras. E, no canto inferior direito, o logo do Comunica_TEA (Fim da Descrição).

Fonte: ComunicaTEA_pais (2020)

Esse recurso de CAA está organizado como um texto escrito e o apoio de símbolos gráficos sobre o processo para participar do jogo Cara a Cara. Com base em Beukelman e Light (2020), depreende-se que a CAA nessa situação favorece a compreensão sobre as etapas ou ações envolvidas no encadeamento do jogo. A partir desse recurso, um estudante com NCC e seus parceiros de comunicação podem coordenar melhor suas ações para interagir ao jogar. A interação comunicativa promovida pelo jogo e apoiada pelo recurso de CAA favorece não apenas o desenvolvimento do vínculo e da relação, mas também do diálogo interno pois, o usuário pode recorrer visualmente ao recurso para recordar o que é preciso fazer a cada passo. Assim, esse recurso apoia a compreensão, aumenta a participação e, de acordo com as habilidades de cada usuário, pode apoiar também o desenvolvimento da linguagem.

A segunda categoria com maior incidência, Orientações sobre o uso de CAA, voltouse a conteúdos destinado à instrumentalização dos parceiros de comunicação acerca do uso da CAA. Sabe-se que a comunicação eficiente requer a construção conjunta de mensagens e sentidos (BEUKELMAN; LIGHT, 2020), portanto, os parceiros de comunicação, quer pais, familiares, e todos os que mantêm interações com os estudantes com NCC necessitam 
também de apoio quanto ao uso da CAA, ainda mais nesse momento, quando as relações familiares se intensificam devido ao maior tempo de permanência em casa e também em função das diferentes dinâmicas interacionais mediadas pelas mídias no Ensino Remoto Emergencial. De fato, quanto mais orientação e apoio os parceiros recebem, melhores são as perspectivas de qualidade nas interações promovidas por todo tipo de atividade, educacional ou não. A Figura 2, é um exemplo de postagem com ênfase na mediação comunicativa, ou seja, relativa ao desenvolvimento de uma interação produtiva entre os usuários de CAA e os parceiros de comunicação.

Figura 2 - Exemplo da categoria Orientações sobre o uso de CAA.
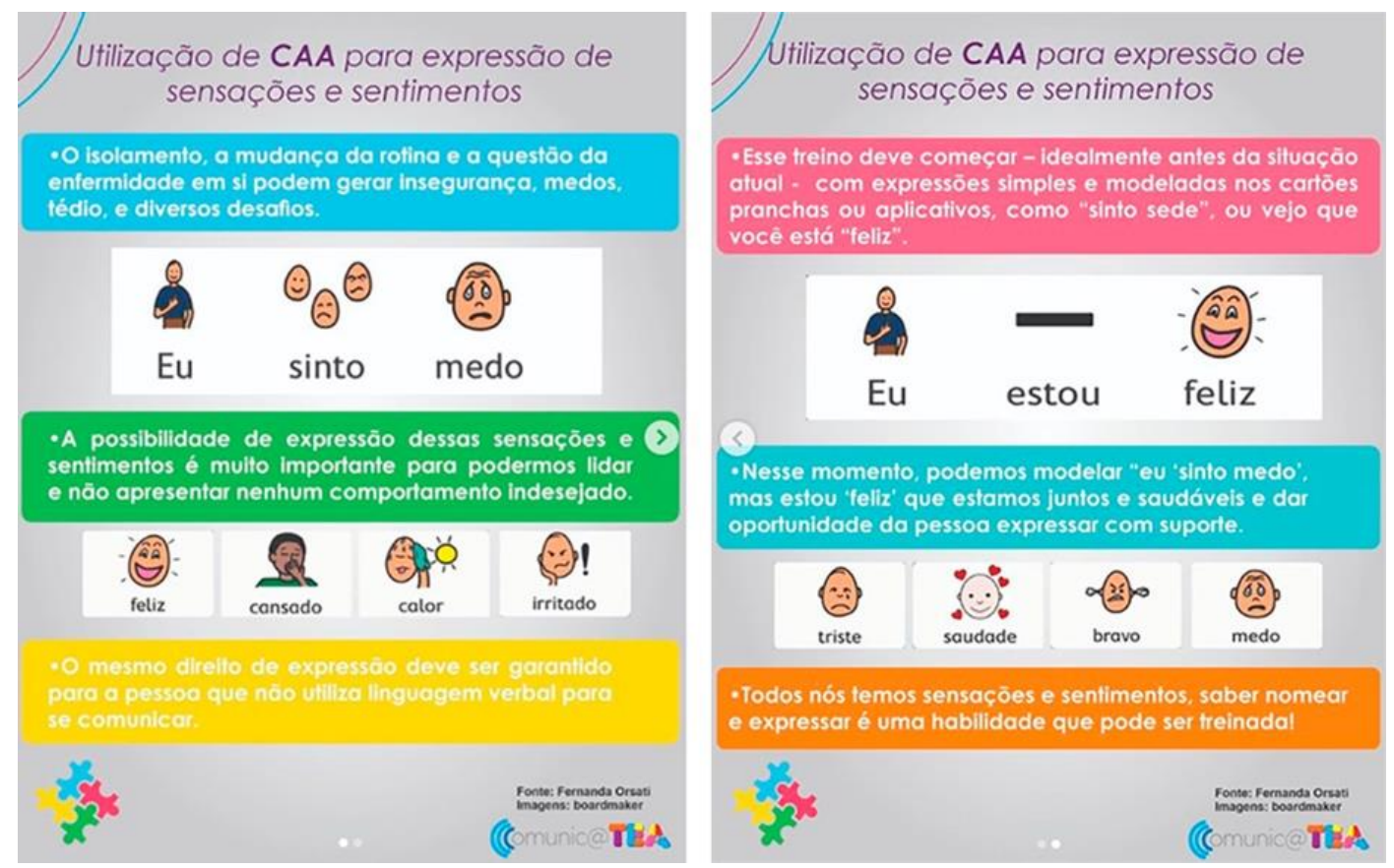

(Descrição da Imagem) Figura composta por 2 imagens verticais da página ComunicaTEA pais. Sobre fundo cinza claro retângulos brancos com símbolos e palavras abaixo de cada símbolo. Antes e depois de cada retângulo branco, um retângulo colorido com texto em branco. No topo de cada imagem o título em roxo: Utilização de CAA para expressão de sensações e sentimentos. E, no canto inferior direito, o logo do Comunica_TEA (Fim da Descrição).

Fonte: ComunicaTEA_pais (2020)

A Figura 2 oferece um conteúdo para conscientizar e orientar parceiros de comunicação quanto ao gerenciamento emocional de usuário de CAA, considerando esse momento de inquietação e incerteza devido à Covid-19, trata-se de uma orientação e recurso importante a ser aplicado para diminuir a frustração e problemas de comportamento.

A categoria Atividade Educativa Formal englobou recursos de CAA com ênfase em conteúdos acadêmicos (português, matemática, ciências humanas e ciências naturais) e leitura (Figura 3). 
Figura 3 - Exemplo da categoria Atividade Educativa Formal.
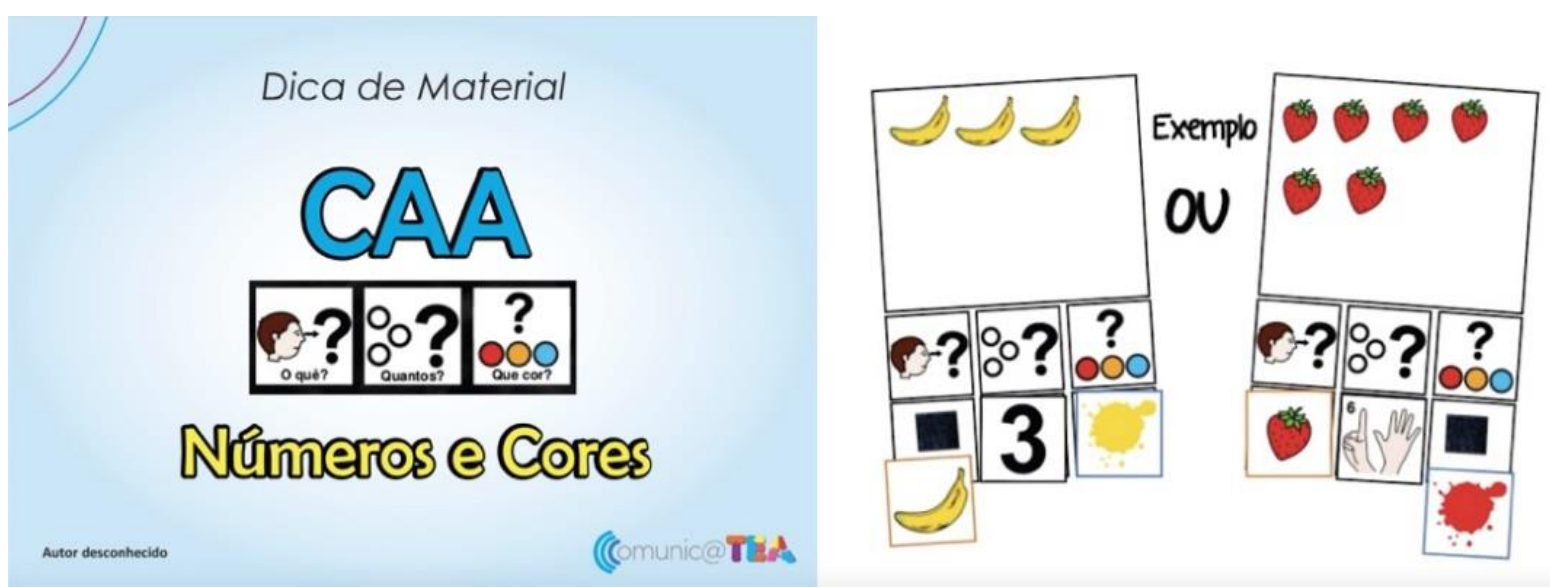

(Descrição da Imagem) Figura composta por 2 imagens horizontais da página ComunicaTEA_pais. A primeira, sobre fundo azul claro, no topo o texto em preto: dica de material, e no centro, 3 quadrados de fundo branco com um símbolo e uma palavra em cada. Acima deles, em azul e bem grande CAA. E abaixo deles, em amarelo: números e cores. E, no canto inferior direito, o logo do Comunica_TEA (Fim da Descrição).

Fonte: ComunicaTEA_pais (2020)

Esse exemplo ilustrativo evidencia como a CAA pode ser integrada às atividades de apoio à aprendizagem. Nesse caso, a CAA facilita a compreensão das informações, a resposta do usuário de CAA com o uso de símbolos concretos, ou seja, imagens que se parecem com o que representam e, portanto, aumentam a participação. Nessa situação, além da aprendizagem de conceitos ligados à matemática (números e quantificação), os recursos de CAA podem certamente auxiliar no desenvolvimento da comunicação - da habilidade comunicativa para responder perguntas - e, da linguagem, com o vocabulário de alimentos e cores nessa instância.

Para exemplificar um conteúdo da categoria COVID-19, a figura 4 é apresentada. Essa postagem contém um conjunto de recursos de CAA, (1) explica o que é a COVID-19 e oferece informações sobre como evitar o contágio, (2) apresenta um texto apoiado por símbolos e (3) uma prancha de escolhas para que o usuário possa definir suas atividades nesse período de distanciamento social. Esses materiais certamente são bem relevantes e têm aplicabilidade para uma variedade de interações nesse momento de isolamento social. 
Figura 4 - Exemplo da categoria COVID-19
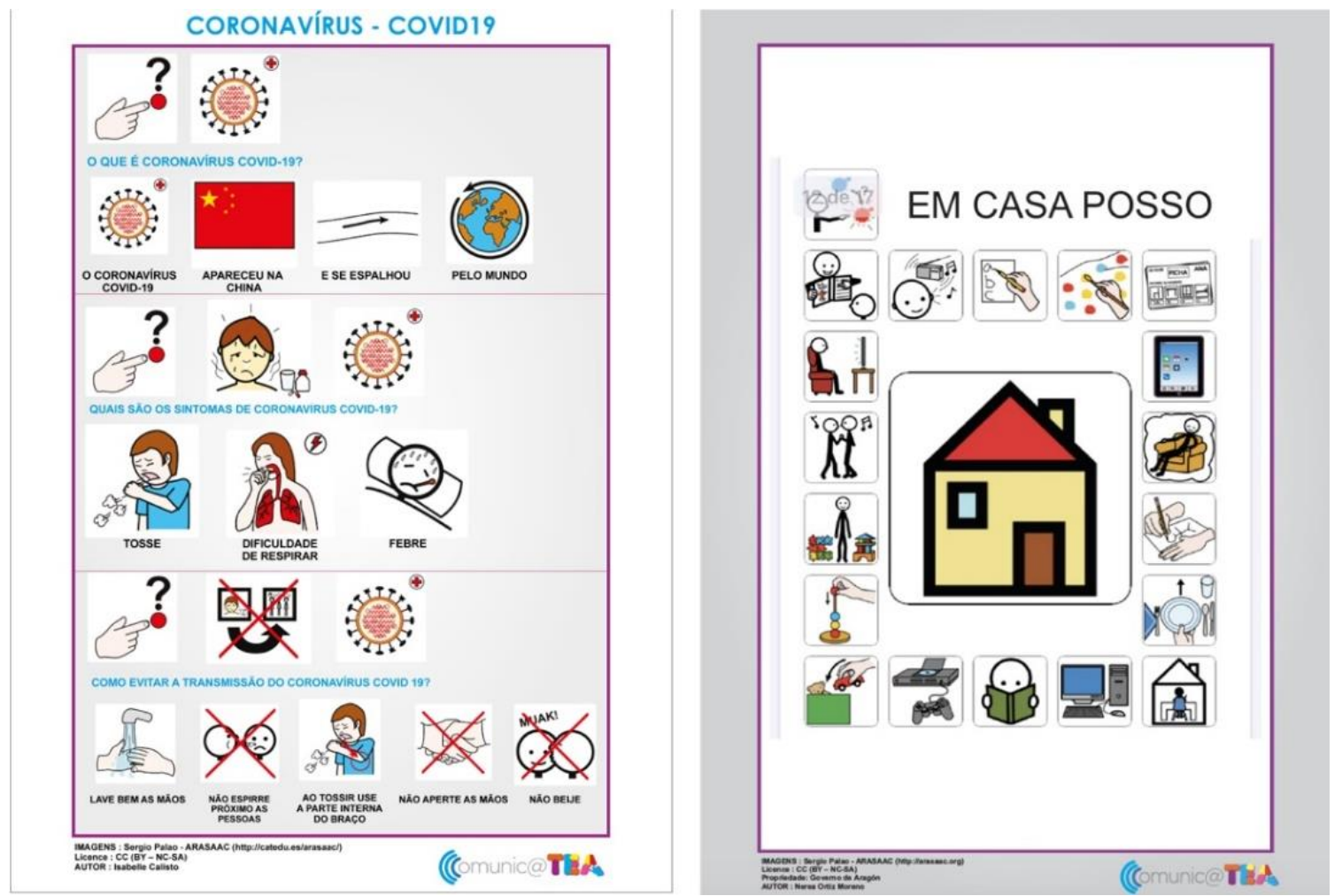

(Descrição da Imagem) Figura composta por duas imagens verticais da página ComunicaTEA_pais. Na primeira, à esquerda, no topo o título em azul claro: Coronavírus - COVID19. Sobre fundo cinza claro com fina borda roxa, 6 linhas com quadrados brancos com 1 símbolo em cada quadrado. Em cada linha, abaixo dos símbolos uma linha de texto em azul claro. E, no canto inferior direito, o logo do Comunica_TEA. Na segunda imagem, à direita, sobre fundo branco 18 símbolos em quadrados brancos contornam um grande símbolo de uma casa no centro da imagem. No topo, em preto: em casa posso. Ao redor da imagem uma fina borda roxa seguida de larga borda cinza clara. E, no canto inferior direito, o logo do Comunica_TEA (Fim da Descrição).

Fonte: ComunicaTEA_pais (2020)

A oferta desses recursos com o apoio de símbolos de CAA favorece dinâmicas mais produtivas no seio da família e apoia a comunicação de pais com filhos com NCC. Por meio desses é possível comunicar necessidades e vontades; passar informações sobre a Covid-19, sobre formas de prevenção e cuidados, apoiando a compreensão e favorecendo a organização por meio do diálogo interno, envolvendo ações que unem estudantes e familiares de modo a permitir que a comunicação e integração do estudante com deficiência seja consolidada em casa com auxílio da CAA. Os recursos apresentam uma explicação sobre a interrupção das aulas presenciais, o que é extremamente relevante, pois a falta deste tipo de orientação e suporte visual pode refletir no comportamento inadequado de crianças e adolescentes com NCC ao não compreenderem o que está acontecendo e as razões associadas ao distanciamento social. Por uma questão de espaço e relevância, não é apresentada figura para a categoria 
Recursos Tecnológicos. Nessa categoria foram identificadas postagens com indicação de aplicativos em geral e aplicativos de CAA. Considera-se que tais indicações estabelecem uma abertura para outros recursos que podem ser de apoio a atividades no período de distanciamento social cumprindo finalidades diversas.

Os estudantes com NCC que estão afastados das atividades escolares nesse momento e suas famílias carecem de apoio e organização para dar seguimento aos processos de desenvolvimento global de seus filhos. Os materiais da página ComunicaTEA_pais favorecem que esse público possa compreender mais e se expressar em uma gama variada de atividades e situações, o que concorre para sua inclusão e participação social e o desenvolvimento de autodeterminação.

\section{Considerações finais}

Muitos usuários de CAA, em especial, aqueles no Ensino Fundamental, estão em um momento de construção da linguagem e expansão de habilidades comunicativas. A disponibilidade de recursos, atividades, orientações e materiais em geral envolvendo o uso de CAA podem servir de amparo a esse processo. A CAA media a comunicação na limitação ou ausência da fala, portanto, as atividades propostas na página ComunicaTEA_pais fornecem oportunidades de aprendizagem e desenvolvimento por meio de materiais para compreender o entorno, estimular a autonomia, fazer escolhas, se engajar em jogos, brincadeiras e uma diversidade de outras atividades. Essas atividades podem suscitar interações ricas, relevantes e significativas para estudantes com NCC e seus parceiros de comunicação, pois têm o potencial para apoiar desde os aspectos mais simples do cotidiano e atividades de lazer para ocupar o tempo de forma produtiva até atividades ligadas a conteúdos escolares.

A página ComunicaTEA_pais, apesar do pouco tempo de existência, fornece uma gama ampla e variada de materiais inerentes à área de CAA em português. Os arquivos das postagens se encontram disponíveis para acesso gratuito através de um link disponível na página. Nesse momento de isolamento social e ERE, o alcance desses conteúdos não pode ser suficientemente estimado. Igualmente, por ser um conteúdo aberto, é mais facilmente acessado por profissionais da educação e saúde que, por sua vez, podem vir a se tornar multiplicadores para tantos outros beneficiários.

Os estudantes com NCC que estão afastados das atividades escolares nesse momento e suas famílias carecem de apoio e organização para dar seguimento aos processos de desenvolvimento global de seus filhos. Os recursos de CAA atendem esse público de modo 
que possa compreender mais e se expressar em uma gama variada de atividades e situações, o que concorre para sua inclusão e participação social e o desenvolvimento de autodeterminação.

Enquanto trabalhos futuros, pretende-se buscar outras fontes de materiais, tanto nacionais quanto internacionais, para aprofundamento e análise dos conteúdos disponibilizados aos estudantes com NCC segundo essas diferentes fontes de oferta.

\section{REFERÊNCIAS}

AMERICAN SPEECH-LANGUAGE-HEARING ASSOCIATION [ASHA]. Roles and responsibilities of speech-language pathologists with respect to augmentative and alternative communication. Technical report. ASHA Supplement 24, 2004.

BARDIN, L. Análise de conteúdo. Trad. Luís de Antero Rego e Augusto Pinheiro. Lisboa: Edições 70, 2011.

BONOTTO, R. C. S. Uso da comunicação alternativa no autismo: um estudo sobre a mediação com baixa e alta tecnologia. 2016. $180 \mathrm{f}$. Tese (Doutorado em Informática na Educação) - Universidade Federal do Rio Grande do Sul, Porto Alegre, 2016.

BRASIL. Lei n. 13.005, de 25 de junho de 2014. Aprova o Plano Nacional de Educação (PNE) e dá outras providências. Diário Oficial da União, Brasília, Edição Extra, Seção 1, p. 1, 26 jun. 2014. Disponível em: http://www.planalto.gov.br/ccivil_03/_ato20112014/2014/lei/113005.htm. Acesso em: maio 2020.

BRASIL. Lei n. 13.146, de 6 de julho de 2015. Institui a Lei Brasileira de Inclusão da Pessoa com Deficiência (Estatuto da Pessoa com Deficiência). Disponível em: http://www.planalto.gov.br/ccivil_03/_Ato2015-2018/2015/Lei/L13146.htm. Acesso em: maio 2020.

BRASIL. Medida Provisória n. 934, de 1 de abril de 2020. Estabelece normas excepcionais sobre o ano letivo da educação básica e do ensino superior decorrentes das medidas para enfrentamento da situação de emergência de saúde pública de que trata a Lei no 13.979 , de 6 de fevereiro de 2020. Diário Oficial da União, Brasília, Edição 63-A, Seção 1 Extra, p. 1, 01 abr. 2020c. Disponível em: http://abre.ai/bgvH. Acesso em maio 2020.

BRASIL. Portaria n. 343, de 17 de março de 2020. Dispõe sobre a substituição das aulas presenciais por aulas em meios digitais enquanto durar a situação de pandemia do Novo Coronavírus - COVID-19. Diário Oficial da União, Brasília, Edição 53, Seção 1, p. 39, 18 mar. 2020a. Disponível em: http://abre.ai/bgvB. Acesso em: maio 2020.

BRASIL. Portaria n. 544, de 16 de junho de 2020. Dispõe sobre a substituição das aulas presenciais por aulas em meios digitais, enquanto durar a situação de pandemia do novo coronavírus - COVID-19, e revoga as Portarias MEC no 343, de 17 de março de 2020, no 345, de 19 de março de 2020, e no 473, de 12 de maio de 2020. Diário Oficial da União, 
Brasília, Edição 114, Seção 1, p. 62, 17 jun. 2020b. Disponível em: https://cutt.ly/9inmB8v. Acesso em: jun. 2020.

BUEKELMAN, D. R.; LIGHT, J. C. Augmentative \& alternative communication: supporting children and adults with complex communication needs. 5. ed. Baltimore: Brookes, 2020.

CASTAMAN, A. S.; RODRIGUES, R. A. Educação a Distância na crise COVID-19: um relato de experiência. Research, Society and Development, v. 9, n. 6, e180963699, 2020.

GARCIA, M. F. et al. Novas competências docentes frente às tecnologias digitais interativas. Rev. Teoria e Prática da Educação, v. 14, n. 1, p. 79-87, jan./abr. 2011.

KOZINETS, R. V. Netnografia: realizando pesquisa etnográfica online. Trad. Daniel Bueno. Porto Alegre: Penso, 2014. 203 p.

MINISTÉRIO DA EDUCAÇÃO [MEC]. A Consolidação da Inclusão Escolar no Brasil 2003 a 2016. Disponível em: http://www.ufpb.br/cia/contents/manuais/a-consolidacao-dainclusao-escolar-no-brasil-2003-a-2016.pdf. Acesso em: 15 mar. 2016.

MOREIRA, J. A.; SCHLEMMER, E. Por um novo conceito e paradigma de educação digital onlife. Revista UFG, v. 20, 2020.

NÓVOA. A. Formação de professores em tempo de pandemia. 1 vídeo (60 min), jun. 2020. Youtube. Disponível em: https:/www.youtube.com/watch?v=ef3YQcbERiM. Acesso em: jun. 2020.

ORGANIZAÇÃO DAS NAÇÕES UNIDAS [ONU]. Convenção das Nações Unidas sobre os Direitos das Pessoas com Deficiência. New York: 2007.

PRATES, D. COVID-19 e o apagamento da pessoa com deficiência. Justificando, 3 abril 2020a. Disponível em: https://www.justificando.com/2020/04/03/covid-19-e-o-apagamentoda-pessoa-com-deficiencia/. Acesso em: jun. 2020.

PRATES, D. O contágio pelo Coronavírus para pessoas com deficiência. Justificando, 24 março 2020b. Disponível em: https://www.justificando.com/2020/03/24/o-contagio-pelocoronavirus-para-pessoas-com-deficiencia/. Acesso em: jun. 2020.

SAMPIERI, R. H.; COLLADO, C. F.; LUCIO, M. B. Metodologia de pesquisa. 5. ed. Porto Alegre: Penso, 2013.

SANTOS, B. S. A cruel pedagogia do vírus. Coimbra: Almedina, 2020.

VIGOTSKI, L. S. A defectologia e o estudo do desenvolvimento e da educação da criança anormal. Educ. Pesqui., São Paulo, v. 37, n. 4, dez. 2011. Disponível em:

http://www.scielo.br/scielo.php?script=sci_arttext\&pid=S1517-

97022011000400012\&lng=en\&nrm=iso. Acesso em: mar. 2014. DOI:

http://dx.doi.org/10.1590/S1517-97022011000400012 
VILLAS BÔAS, L.; UNBEHAUM, S. (Coor.). Educação escolar em tempos de pandemia. Informe 1. Fundação Carlos Chagas, 2020. Disponível em: http://abre.ai/bgvP. Acesso em: jun. 2020.

WILLIAMSON, B.; EYNON, R.; POTTER, J. Pandemic politics, pedagogies and practices: digital technologies and distance education during the coronavirus emergency. Learning, Media and Technology, v. 45, n. 2, p. 107-114, 2020.

WORLD BANK [WB]. Every learner matters: unpacking the learning crisis for children with disabilities. World Bank Group, Washington, DC, 2019. Disponível em:

https://openknowledge.worldbank.org/handle/10986/31946. Acesso em: jun. 2020.

ZHAO, Y. COVID-19 as a catalyst for educational change. Prospects, 2020. Disponível em: https://link.springer.com/article/10.1007\%2Fs11125-020-09477-y. Acesso em: jun. 2020. DOI: https://doi.org/10.1007/s11125-020-09477-y 2020

\section{Como referenciar este artigo}

BONOTTO, R.; CORRÊA, Y.; CARDOSO, E.; MARTINS, D. S. Oportunidades de aprendizagem com apoio da Comunicação Aumentativa e Alternativa em tempos de COVID19. Revista Ibero-Americana de Estudos em Educação, Araraquara, v. 15, n. 4, p. 17301749, out./dez. 2020. e-ISSN: 1982-5587. DOI: https://doi.org/10.21723/riaee.v15i4.13945

Submetido em: 08/04/2020

Revisões requeridas em: 30/05/2020

Aprovado em: 01/07/2020

Publicado em: 30/08/2020 
RIAEE - Revista Ibero-Americana de Estudos em Educação, Araraquara, v. 15, n. 4, p. 1730-1749, out./dez. 2020. e-ISSN: $1982-5587$. DOI: https://doi.org/10.21723/riaee.v15i4.13945 